\title{
The Functional Tests after ACL Reconstruction with and without Meniscal Repair
}

\author{
Sineenad Janewanitsataporn, M.D.
}

Department of Physical Medicine and Rehabilitation, Suratthani Hospital, Mueang, Suratthani 84000, Thailand.

Received 26 August 2019 • Revised 29 November 2019 • Accepted 22 December 2019 • Published online 20 February 2020

\section{Abstract:}

Objective: This study aims to compare the functional tests after anterior cruciate ligament reconstruction (ACLR) with and without meniscal repair after the rehabilitation program and before returning to sports.

Material and Methods: Patients who underwent ACLR using hamstring tendon autograft during 2016-2017 were invited to participate in this study and divided into 2 groups including with and without meniscal repairs according to the surgical reports. A group of ACLR with meniscal repair required the conservative anterior cruciate ligament (ACL) rehabilitation program because the non-weight-bearing approach should be applied during the first 6 weeks. These patients were followed up clinically until the rehabilitation program finished. The functional tests were applied with the 4 single-leg hop tests, as reported in the limb symmetry index (LSI).

Results: Forty-three patients were enrolled in the program. These patients were divided into 2 groups: ACLR with meniscal repair $(n=20)$ and ACLR without meniscal repair $(n=23)$. It was found that there was no statistically significant difference between the two groups with respect to duration for completing the rehabilitation program ( $p-v a l u e=0.38)$. Also, there was no statistically significant difference between the two groups with respect to the LSI of all 4 singleleg hop tests of duration for completing the rehabilitation program.

Conclusion: The conservative ACL rehabilitation program after meniscal repair surgery did not affect the function tests in terms of duration for completing the rehabilitation program before return to sports (RTS).

Keywords: ACLR, functional tests, limb symmetry index, meniscal repair, rehabilitation

Contact: Department of Physical Medicine and Rehabilitation, Suratthani Hospital, Mueang, Suratthani 84000, Thailand.

E-mail: c.sineenad@hotmail.com

() 2020 JHSMR. Hosting by Prince of Songkla University. All rights reserved.

This is an open access article under the CC BY-NC-ND license

(http://www.jhsmr.org/index.php/jhsmr/about/editorialPolicies\#openAccessPolicy).
J Health Sci Med Res 2020;38(2):73-79 doi: 10.31584/jhsmr.2020726 www.jhsmr.org 


\section{Introduction}

The anterior cruciate ligament $(A C L)$ is the most commonly injured knee ligament which inflicts concomitant injuries to the meniscus accounted for 64.0 to $77.0 \%$ of individuals who suffer the ACL rupture and require surgical intervention. ${ }^{1}$ Surgeons can perform the arthroscopic anterior cruciate ligament reconstruction (ACLR) along with meniscal surgery. The meniscal surgery options include meniscectomy and meniscal repair. The post-operative management of the ACLR with meniscal repair requires more conservative approach than the ACLR with meniscectomy to ensure the successful treatment outcomes. ${ }^{2}$ The rehabilitation program, after ACLR with meniscectomy, does not require a modification of the standard ACL rehabilitation program. On the other hand, the rehabilitation program after ACLR with meniscal repair does require a modified $\mathrm{ACL}$ rehabilitation program, because the non-weightbearing approach should be applied during the first 6 weeks in order to better protect the repair and improve the healing process. Unfortunately, this process causes usually significant muscle atrophy and muscle inhibition during the non weight bearing period, especially in the quadriceps muscle. According to non-weight bearing studies, it was found that the vastus medialis muscles were the first part to atrophy. A systematic review demonstrated quadriceps activation failure was commonly found among patients after ACLR. ${ }^{3}$ Quadriceps strength and endurance are very important factors for normal knee joint function. Hence, restoring normal quadriceps function after knee joint injuries is an essential element of rehabilitation. The conservative ACL rehabilitation program with non-weight-bearing approach during the first 6 weeks following surgery might affect the quadriceps strength and the functional abilities of patients' leg.

After the ACLR and rehabilitation, clinical tests including strength testing and laxity measurements does not correlate well with the functional abilities. The functional tests were developed to evaluate surgical and therapeutic outcomes. $^{4,5}$ The 4 single-leg hop tests have frequently been proposed as a practical performance-based outcome measurement that reflects the integrated effect of neuromuscular control strength and confidence in the limb. ${ }^{6}$ Measurements are performed on both extremities so that test performance on the operated limb and the other limb can be expressed the value as a percentage on the basis of the so-called limb symmetry index (LSI). The LSI was developed by Noyes et al. ${ }^{7}$, so as to evaluate the difference between two legs in the functional testing. The LSI $>85.0 \%$ indicates normal limb symmetry and an abnormal function test score represents a serious risk of physical limitations during sports activities.

The purpose of this study is to compare the functional tests after ACLR both presence and absence of meniscal repair in terms of duration for completing the rehabilitation program and before return to sport (RTS).

This research investigated the potential additive effect of meniscal repair on post ACLR functional abilities. Data from this investigation was clinical outcomes which could determine whether a recovery-focused rehabilitation program should be developed for individuals with meniscal repair surgery.

\section{Material and Methods \\ Participants}

Patients who had undergone an arthroscopic ACLR with hamstring tendon autografts during 2016-2017 were invited to participate in this study (Table 1). All surgeons utilized a similar surgical technique (anteromedial portal drilling, tibial screw fixation and femoral endobutton fixation). Patient aged between 15 to 50 years old. Patients would be excluded if they had a previous history of surgery (other than $\mathrm{ACL}$ reconstruction) to either knee, had suffered a previous $\mathrm{ACL}$ injury or had a known heart condition. Pregnant females were also excluded. Surgical reports were 
obtained in order to report any concomitant meniscal damage that required surgical intervention (Table 1). Details of the post-operative home-based rehabilitation program are illustrated in Appendix 1.,9 All patients completed standard post-operative rehabilitation at the Suratthani rehabilitation outpatient clinic which included seven stages that began during the first post-operative week. Each stage was assigned for evaluating the patients who could complete the rehabilitation goals successfully. The duration for completing a program lasts at least six months after surgery. In general, the ACL rehabilitation program emphasizes full knee extension range of motion immediately and knee flexion including tolerated and progression of functional exercises, quadriceps and other leg muscles exercises, proprioception, balance, neuromuscular control and sport specific exercises. Variations occurred in the ACL rehabilitation programs are based on meniscal repair surgery. A group of ACLR with meniscal repair requires a modified conservative ACL rehabilitation program offering non- weight-bearing suggest on for the first 6 weeks. This study has been approved by the Regional Ethics Committee of Suratthani Hospital.

\section{The 4 single-leg hop tests}

After completing a rehabilitation program and before RTS, the patients were examined with a series of 4 single-leg hop tests. The 4 single-leg hop tests included the single-leg hop for distance, triple hop for distance, crossover triple hop for distance over a single line and continuous 6-meter timed hop with maximum effort. ${ }^{10}$ The diagram for each hop test is shown in Figure 1. The participants were required to stick their landing to be successful in all the tests. The measurements were performed on both extremities so that test performance on the operated limb and the other limb can be expressed the value as a percentage on the basis of the so-called LSI.

LSI (\%)=injured leg/uninjured leg $\times 100$

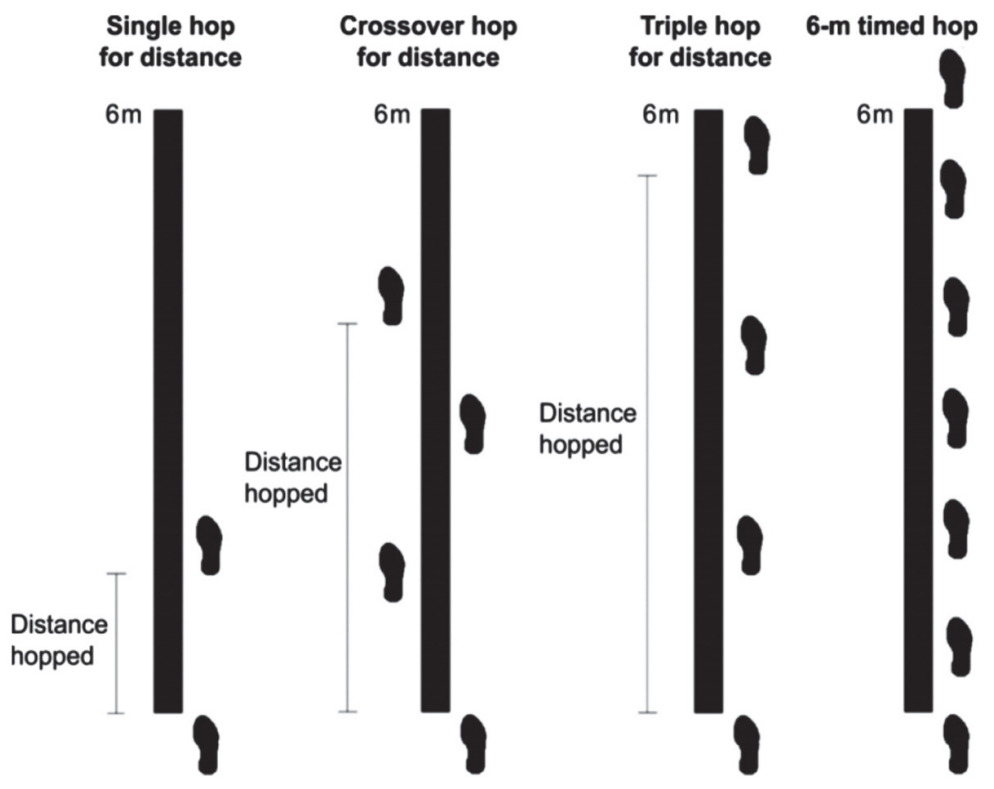

$\mathrm{m}=$ meter

Figure 1 The 4 single-leg hop tests: single hop for distance, crossover hop for distance, triple hop for distance, and 6 meter timed hop ${ }^{11}$ 


\section{Statistical analysis}

Data was expressed as mean \pm standard deviation. A t-test was used to compare the groups. Statistical analyses were conducted using STATA version 12.0 (Stata Corp, College Station, TX). A p-value of less than 0.05 was considered statistically significant.

According to Polit \& Hungler, 1995; the semi experimental research's sample size consideration required at least 20-30 members for each group. ${ }^{12}$

\section{Results}

\section{Subject demographics}

Forty-three patients were enrolled in the program. These patients were divided into 2 groups: ACLR with meniscal repair $(n=20)$ and ACLR without meniscal repair $(n=23)$. The median age and body mass index $(B M I)$ were not different between the 2 groups ( $p$-value=0.86, 0.86). The preoperative sport activity level was significant difference between the 2 groups ( $p$-value=0.04) (Table 1). The ACLR group with meniscal repair had 8 athletic patients $(40.0 \%)$, whilst the ACLR group without meniscal repair had 3 athletic patients $(13.0 \%)$.

\section{Duration for completing the rehabilitation} program

Mean value of duration for completing the rehabilitation program and before RTS in the group of ACLR with meniscal repair was 32.2 weeks and in the group of ACLR without meniscal repair was 34.3 weeks. Therefore, there was no statistically significant difference between the 2 groups in terms of duration for completing the rehabilitation program ( $p$-value $=0.32$ ) (Table 1).

Table 1 Participants demographic data

\begin{tabular}{lll}
\hline Demographic data & $\begin{array}{l}\text { ACLR with meniscal repair } \\
(\mathbf{n = 2 0})\end{array}$ & $\begin{array}{l}\text { ACLR without meniscal repair } \\
(\mathbf{n = 2 3})\end{array}$ \\
\hline Sex & Male=20, Female=0 & $\begin{array}{l}\text { Male=21, Female=2 } \\
\text { P-value }\end{array}$ \\
Age (years) & $26.40 \pm 9.24^{*}$ & $26.91 \pm 9.66^{*}$ \\
BMI $\left(\mathrm{kg} / \mathrm{m}^{2}\right)$ & $23.47 \pm 3.52^{*}$ & $23.67 \pm 6.37^{*}$ \\
Preoperative sport activity level & Amateur=12, Athlete=8 & Amateur=20, Athlete=3 \\
Duration for completing program (weeks) & $32.2 \pm 6.57^{*}$ & $34.3 \pm 7.02^{*}$ \\
LSI $>85.0 \%$ & $18(90.0 \%)$ & $21(91.3 \%)$ \\
\hline
\end{tabular}

ACLR=anterior cruciate ligament reconstruction, BMl=body mass index, LSI=limb symmetry index

*mean \pm standard deviation

Table 2 Limb symmetry index for each single hop test and all 4 combined

\begin{tabular}{llll}
\hline LSI & $\begin{array}{l}\text { ACLR with } \\
\text { meniscal repair }\end{array}$ & $\begin{array}{l}\text { ACLR without } \\
\text { meniscal repair }\end{array}$ \\
\hline Single hop LSI & $89.58 \pm 6.80^{*}$ & $92.74 \pm 6.37^{*}$ & 0.12 \\
Triple hop LSI & $93.20 \pm 6.96^{*}$ & $93.64 \pm 5.79^{*}$ & 0.82 \\
Crossover hop LSI & $94.00 \pm 4.93^{*}$ & $94.55 \pm 5.84^{*}$ & 0.74 \\
Timed hop LSI & $89.10 \pm 8.45^{*}$ & $92.90 \pm 8.08^{*}$ & 0.14 \\
All tests LSI & $91.47 \pm 4.56^{*}$ & $93.46 \pm 5.08^{*}$ & 0.19 \\
\hline
\end{tabular}

ACLR=anterior cruciate ligament reconstruction, LSI=limb symmetry index

*mean \pm standard deviation 


\section{The 4 single-leg hop tests}

The patients had all test LSI value $>85.0 \%$. There were 18 patients in the ACLR with meniscal repair group (90.0\%) and 21 patients in the ACLR without meniscal repair group (91.3\%). According to the number of patients achieving LSI $>85.0 \%$ in all tests, there was no statistically significant difference between the two groups ( $p$-value $=0.88)$ (Table 1). In addition, the LSI of all 4 single-leg hop tests showed no significant difference between the 2 groups ( $p$-value=0.19) (Table 2).

\section{Discussion}

This study was conducted in order to compare the function test results after ACLR with meniscal repair with the conservative ACL rehabilitation program for nonweight bearing during the first 6 weeks and ACLR without meniscal repair with the standard $A C L$ rehabilitation program before RTS. The most important finding indicated that both groups of patients had no significant differences in terms of duration for completing the rehabilitation program before RTS. Thus, the non-weight bearing approach during first 6 weeks mostly slowed down the initial stages of the ACL rehabilitation program. However, it did not significantly prolong the whole program. Similar results were previously reported by Lepley. ${ }^{1}$ There was no difference between the 2 groups in ACLR only and ACLR with meniscal surgery in terms of the duration of time until return to sport. Nevertheless, the means value of duration for completing the rehabilitation program in the ACLR with meniscal repair group (32.2 weeks) was shorter than the ACLR without meniscal repair group (34.3 weeks). The reason for this shorter duration could be explained by the percentage of athletes, as the group of ACLR with meniscal repair had more athletic patients $(40.0 \%)$ than the group of ACLR without meniscal repair (13.0\%). Additionally, these athletic patients had every intention of making a full recovery, and fully cooperated with the rehabilitation program, as they wanted to return to their respective sport as soon as possible.

The ACL rehabilitation program had seven stages in total. Patients would complete the rehabilitation goals by passing each single stage. The ACLR patients with meniscal repair were limited to the $2^{\text {nd }}$ and $3^{\text {rd }}$ stages activities (step up, toes rise, wall slide and standing single leg) because of their non-weight bearing period. They, subsequently, could be involved in all activities in stage 4 (6 weeks after operation). Most of the patients had slow progress of the program at stage 5 if they did not join in the training program regularly, they could not pass the single leg squat test (SLST). The SLST was assigned to challenge the neuromuscular control and strength of the surgical limb. ${ }^{13}$ All patients in the ACLR with meniscal repair group could undergo an intensive training to achieve better strength and proprioceptionas same as the patients in the non-meniscal repair group in this stage. Passing stage 5 meant those patients had good SLST then they could train a sport specific program (stage 6-7).

After completing a rehabilitation program and before RTS, the patients were examined with the function tests outcomes, so-called LSI. The LSI values of all 4 single-leg hop tests were not different between the 2 groups. Also, the number of patients who achieved LSI $>85.0 \%$ in all tests was not different between the 2 groups. This finding is in agreement with Shelbourne and colleagues $^{14}$ who found that there was no difference in quadriceps strength and single-leg hop performance tests of 5 to 15 years post-surgery in individuals who suffered meniscectomy and/or articular cartilage damage as compared to isolated ACLR. Eric and colleagues ${ }^{15}$ found that the presence of concomitant injuries was not associated with the recovery of muscle function at the one-year follow-up after ACLR.

The patients had muscle inhibition during 6 weeks for non-weight-bearing period. Their problem could be 
solved by intensive training strength, balance and neuromuscular control. In whole rehabilitation program, they could achieve normal LSI. Thus, the effective rehabilitation programs could promote good quadriceps function for long-term healthy knee joint.

\section{Conclusion}

The conservative ACL rehabilitation program after meniscal repair surgery did not affect the function tests in terms of duration for completing the rehabilitation program before RTS.

\section{Acknowledgement}

The author thanks biostatistician Thidajit Maneewat, Ph.D. and Sopida Khoynueng for help with statistical analyses and also the Department of Orthopaedics at Suratthani Hospital.

\section{Conflict of interest}

No potential conflicts of interest relevant to this article were reported.

\section{References}

1. Lepley LK, Wojtys EM, Palmieri-Smith RM. Does concomitant meniscectomy or meniscal repair affect the recovery of quadriceps function post ACL reconstruction?. Knee Surg Sports Traumatol Arthrosc 2015;23:2756-61.

2. Cavanaugh JT, Killian SE. Rehabilitation following meniscal repair. Curr Rev Musculoskelet Med 2012;5:46-58.

3. Hart JM, Pietrosimone B, Hertel J, Ingersoll CD. Quadriceps activation following knee injuries: a systematic review $\mathrm{J}$ Athl Train 2010;45:87-97.

4. Sueyoshi T, Nakahata A, Emoto G, Yuasa T. Single-leg hop test performance and isokinetic knee strength after anterior cruciate ligament reconstruction in athletes. Orthop $\mathrm{J}$ Sports Med 2017;5. doi: 10.1177/2325967117739811.

5. Reid A, Birmingham TB, Stratford PW, Alcock GK, Giffin JR.
Hop testing provides a reliable and valid outcome measure during rehabilitation after anterior cruciate ligament reconstruction. Phys Ther 2007;87:337-49.

6. Abrams GD, Harris JD, Gupta AK, McCormick FM, BushJoseph CA, Verma NN, et al. Functional performance testing after anterior cruciate ligament reconstruction a systematic review. Orthop J Sports Med 2014;2. doi: 10.1177/2325967 113518305.

7. Noyes FR, Barber SD, Mangine RE. Abnormal lower limb symmetry determined by function hop tests after anterior cruciate ligament rupture. Am J Sports Med 1991;19:513-8.

8. D’amato M, Bach BR. Knee injuries. In: Brotzman SB, Wilk KE, editors. Handbook of orthopaedic rehabilitation. $2^{\text {nd }}$ ed. Philadelphia: Mosby Elsevier; 2007;p.375-486.

9. Baltaci G, Yilmaz G, Atay AO. The outcomes of anterior cruciate ligament reconstructed and rehabilitated knees versus healthy knees: a functional comparison. Acta Orthop Traumatol Turc 2012;46:186-95.

10. Logerstedt D, Grindem H, Lynch A, Eitzen I, Engebretsen L, Risberg MA, et al. Single-legged hop tests as predictors of self-reported knee function after anterior cruciate ligament reconstruction. Am J Sports Med 2012;40:2348-56.

11. Grindem H, Logerstedt D, Eitzen I, Moksnes H, Axe MJ, Snyder-Mackler $\mathrm{L}$, et al. Single-legged hop tests as predictors of self-reported knee function in non operatively treated individuals with anterior cruciate ligament injury. Am J Sports Med 2011;39:2347-54.

12. Polit DF, Hungler BP. Nursing research: principles and methods. $5^{\text {th }}$ ed. Philadelphia: JB Lippincott; 1995.

13. Hall MP, Paik RS, Ware AJ, Mohr KJ, Limpisvasti O. Neuromuscular evaluation with single-leg squat test at 6 months after anterior cruciate ligament reconstruction. Orthop J Sports Med 2015;3. doi:10.1177/2325967115575900.

14. Shelbourne KD, Gray T. Results of anterior cruciate ligament reconstruction based on meniscus and articular cartilage status at the time of surgery. Five- to fifteen-year evaluations. Am J Sports Med 2000;28:446-52.

15. Senorski EH, Svantesson E, Beischer S, Thomee C, Grassi A, Krupic $F$, et al. Concomitant injuries may not reduce the likelihood of achieving symmetrical muscle function one year after anterior cruciate ligament reconstruction: a prospective observational study based on 263 patients. Knee Surg Sports Traumatol Arthrosc 2018;26:2966-77. 


\section{Appendix 1}

Home-based ACL rehabilitation program ${ }^{8,9}$

\begin{tabular}{ll}
\hline Time frame & Rehabilitation goal \\
\hline Stage1 & ROM full extension \\
Week 0-2 & Quadriceps contraction \\
& Weight bearing as tolerated with crutches \\
& Except meniscal repair group non weight bearing first 6 weeks
\end{tabular}

\section{Activities}

On knee brace full extension all times Isometric quadriceps exercise Ankle pump

SLR (flex, abduct, extend)

Stretching hamstring and gastrocnemius muscle Prone hang, pillow under heel

Gait training

Patellar mobilization

\begin{tabular}{ll}
\hline Stage2 & ROM 0-90 \\
Week 2-4 & Closed chain quadriceps exercise
\end{tabular}

Knee brace $0-90^{\circ}$

PROM 0-90

Heel slide

Prone hamstring exercise

Wall slide knee flex $45^{\circ}$

Stage $3 \quad$ ROM 0-120

Week 4-6 Increased muscle strength and endurance

Enhance propioception, balance, neuromuscular control

Unlocked knee brace

PROM 0-120

Stationary bike

Step up 4"step forward and lateral

Toe rises

Wall slide knee flex $90^{\circ}$

Shift weight, standing single leg

\begin{tabular}{lll}
\hline Stage 4 & ROM 0-140 & PROM 0-140 \\
Week 6-8 & Normal gait pattern & Step up 8"step forward and lateral \\
& Increased muscle strength and endurance & Wall slide knee flex $90^{\circ}$ with weight and ball \\
& With meniscus repaired titrate weight bearing & Wall slide single leg $45-90^{\circ}$ \\
& & Backward walking \\
& & Leg press exercise (0-60 degree) \\
\hline Stage 5 & Full ROM & Trademill walking (flat only) \\
Week 8-12 & Enhance propioception, balance, neuromuscular control & Forward lunge exercise \\
& Good single leg squat test & Wobble board balance two leg \\
& & Single leg squat \\
\hline Stage 6 & Full ROM & Reverse lunge exercise \\
Week 12-18 & Restore functional capability and confidence & Jogging and light running \\
& Good single leg squat test & Knee extension exercise \\
& & Agility exercise \\
\hline Stage 7 & Maintained muscle strength and endurance & Outdoor bike on flat road \\
Week 18-24 & neuromuscular control & Running, jumping, hoping \\
& Return to sport & Agility exercise \\
\hline
\end{tabular}

$\mathrm{ROM}=$ range of motion, $\mathrm{SLR}=$ straight leg raising, $\mathrm{PROM}=$ passive range of motion 